Pacific Journal of Mathematic 


\title{
THE TOPOLOGY OF ALMOST UNIFORM CONVERGENCE
}

\author{
JOHN W. BRACE
}

The theorem of Arzelà [3, 4, 6] (see Theorem 2.2) which gives a necessary condition and a sufficient condition for a net of continuous functions to converge to a continuous function plays an important part in functional analysis. In the case of linear topological spaces it has been observed that the quasi-uniform convergence [3] (see Definition 2.1) which Arzelà presented in his theorem is related to the weak and weak* topologies $[6,9,20]$. With this fact in mind it was surmised that quasi-uniform convergence would present a useful method for topologizing function spaces. This paper presents such a topology and displays some of its properties and applications. The resulting topology will be called the topology of almost uniform convergence.

In $\S 1$ the topology is defined by means of a base for the neighborhood system of the zero function (origin). It should be noted that there is a similarity between the development of uniform convergence topologies [17] and the topology of almost uniform convergence. Section 2 shows that convergence of a net of functions for the topology implies quasi-uniform convergence. A net of functions having the property that every subnet converges quasi-uniformly will converge for the topology. In $\$ 3$ the concept of almost uniform convergence is extended to the case where convergence occurs on each member of a family of subsets of the domain space. Section 4 examines the properties of various function spaces in regard to the topology of almost uniform convergence. In particular, Theorem 4.3 shows that convergence in this topology for a net of bounded continuous functions over a regular Hausdorff space $S$ is equivalent to pointwise convergence of their extensions on the Stone-Cèch compactification of $S$.

Section 5 uses the topology of almost uniform convergence to obtain the weak topology for certain locally convex linear topological spaces. It is necessary in $\S 5$ to modify the topology of almost uniform convergence to form a finer (stronger) topology which is called the topology of convex almost uniform convergence. With this new topology, Theorem 5.6 shows that the weak topology for a function space, which was originally a locally convex linear topological space for a uniform convergence topology, is the topology of convex almost uniform convergence. Theorem 5.9 parallels a theorem in Banach's book (page 134) [5] in giving a necessary and sufficient condition for the weak conver-

Received November 3, 1958. This research was supported by the United States Air Force through the Air Force Office of Scientific Research of the Air Research and Development Command under Contract No. AF 18 (603)-78.

Presented to the Society, October 27, 1956. 
gence of a net (instead of a sequence) from the Banach space of all continuous on the closed unit interval.

In the same manner Theorem 5.10 gives a necessary and sufficient condition for the convergence in the weak topology of a net in $L^{1}$. A similar theorem for sequences can be found in [12; page 89].

1. The almost uniform convergence topology. In establishing the existence of the almost uniform convergence topology for a collection of functions, the first step is to determine a class of sets that can be used to generate the neighborhood system of the zero element of the function space $[7,15,19]$.

1.1 Definition. Let $\mathscr{G}(S, F)$ be a linear space of functions on an abstract set $S$ into a locally convex linear topological space $F$. Then a subset $U$ of $\mathscr{G}(S, F)$ has the property $(\alpha)$ in $\mathscr{G}(S, F)$ if it satisfies the condition: for some neighborhood $V$ of 0 in $F$ it is true that for each finite subset $\left\{f_{i}, \cdots, f_{k}\right\}$ of $\mathscr{G}(S, F) \sim U$ there is an $x$ in $S$ such that $f_{i}(x) \notin V(i=1, \cdots, k)$.

1.2 Lemma. For each subset $U$ of $\mathscr{G}(S, F)$ with property $(\alpha)$, there is a convex circled subset $W$ of $U$ with property $(\alpha)$. Furthermore each $U$ is radial at the origin of $\mathscr{G}(S, F)$ if and only if $f[S]$ is bounded for each $f$ in $\mathscr{Y}(S, F)$.

Proof. Let $V_{0}$ be a convex circled neighborhood of 0 in $F$ whose relationship to $U$ is as stated in property $(\alpha)$. Accepting the Hausdorff minimal principle, there exists a subset of $U$, call it $W$, which is minimal with respect to the property $(\alpha)$ for the previously mentioned neighborhood $V_{0}$ of 0 in $F$.

Observe that a function $f$ is in $W$ if and only if there exists a finite subset $\left\{f_{i}, \cdots, f_{n}\right\}$ of $\mathscr{G}(S, F) \sim W$ such that for every $x$ in $S$ either $f(x)$ is in $V_{0}$ or $f_{j}(x)$ is in $V_{0}$ for at least one $f_{j}, j=1, \cdots, k$. For two functions $f$ and $g$ in $W$ let the two finite sets $\left\{f_{1}, \cdots, f_{m}\right\}$ and $\left\{g_{1}, \cdots, g_{n}\right\}$ bear this relationship to $f$ and $g$ respectively. The union $\left\{f_{1}, \cdots, f_{m}\right.$, $\left.g_{1}, \cdots, g_{n}\right\}$ bears the same relationship to $t f+(1-t) g$ for all $t$ such that $0 \leq t \leq 1$. Thus it is concluded that $W$ is a convex set.

The circularity of $W$ is obtained in a similar manner.

Assume that for each $f$ in $\mathscr{C}(S, F)$ there is a scalar $c$ such that $f[S] \subset c V_{0}$. This implies that $f$ is in $c W \subset c U$. In other words, $U$ is radial at 0 in $\mathscr{C}(S, F)$.

Assuming each $U$ to be radial and the existence of an $f$ in $\mathscr{G}\left(S, F^{\prime}\right)$ such that $f[S]$ is not bounded, there is a sequence $\left\{x_{n}\right\} \subset S$ and a convex circled neighborhood $V$ of the origin in $F^{\prime}$ with the property that $\frac{1}{n} f\left(x_{n}\right)$ 
is not in $V$ for $n=1,2, \cdots$. Let $U_{0}=\mathscr{C}(S, F) \sim\left\{f, \frac{1}{2} f, \frac{1}{3} f, \cdots\right\}$. This is a contradiction because $U_{0}$ has property $(\alpha)$ and is not radial.

1.3 Theorem. Let $\mathscr{C}(S, F)$ be a linear space of functions on an abstract set $S$ into a locally convex linear topological space $F$. Then all the sets of the form $U_{1} \cap \cdots \cap U_{n}$, where $U_{i}$, has property $(\alpha)$ in $\mathscr{C}(S, F)$, form a local base for a locally convex topology. This topology is called the topology of almost-uniform convergence on $S$. Furthermore, it is a linear topology if and only if $f[S]$ is bounded for each $f$ in $\mathscr{S}(S, F)$ and it is a Hausdorff topology if $F$ is Hausdorff.

Proof. The existence of the almost-uniform convergence topology (linear and non-linear) is obtained from Lemma $2.3[7,15,19]$.

If $F$ is Hausdorff and $f$ is a non-zero element of $\mathscr{Y}(S, F)$ then there is a point $x$ in $S$ and a neighborhood $V$ of 0 such that $f(x)$ is not in $V$. Thus $\mathscr{C}(S, F) \sim\{f\}$ has property $(\alpha)$ and the almost uniform convergence topology is Hausdorff.

2. The convergence of nets. The study of almost uniform convergence topologies is partially motivated by quasi-uniform convergence and almost uniform convergence for nets of functions [17]. Now that the topology has been obtained it is time to consider its relationship to the almost uniform convergence of a net of functions.

2.1 Definition. [3, 6, 21]. A net $\left\{f_{\alpha}, \alpha \in D\right\}$ in $\mathscr{Y}(S, F)$ converges to $f_{0}$ quasi-uniformly on $S$ if $\lim f_{\alpha}(x)=f_{0}(x)$ for each $x$ in $S$ and for every neighborhood $V$ of the zero element of $F$ and $\alpha_{0}$ in $D$ there is a finite subset $\left\{\alpha_{1}, \alpha_{2}, \cdots, \alpha_{n}\right\}$ of $D, \alpha_{i} \geq \alpha_{0}, i=1,2, \cdots, n$, such that for every $x$ in $A, f_{\alpha_{i}}(x)-f_{0}(x)$ is in $V$ for at least one $\alpha_{i}, i=1,2, \cdots, n$.

The importance of quasi-uniform convergence stems from the following theorem.

2.2 Theorem (Arzelà) [3, 4, 6]. If a net of continuous functions on a topological space $X$ converges to a continuous limit, then the convergence is quasi-uniform on every compact subset of $X$. Conversely, if the net converges quasi-uniformly on a subset of $X$, the limit is continuous on this subset.

Since a net converges in a topological space if and only if every subnet converges, the following modification of quasi-uniform convergence is the natural thing to expect.

2.3 Definition [19]. A net $\left\{f_{\alpha}, \alpha \in D\right\}$ in $\mathscr{C}(S, F)$ converges almost uniformly to $f_{0}$ on $S$ if and only if every subnet converges quasiuniformly to $f_{0}$ on $A$. 
2.4 THEOREM. Let $\mathscr{G}(S, F)$ be a function space with the topology of almost uniform convergence on $S$. A net $\left\{f_{\alpha}, \alpha \in D\right\}$ in $\mathscr{C}(S, F)$ converges almost uniformly to $f_{0}$ on $S$ if and only if the net converges to $f_{0}$ in the topology.

Proof. Considering $f_{0}$ to be the zero function, it is assumed that the net converges almost uniformly to $f_{0}$ without being eventually in a set $U$ with property $(\alpha)$. This gives a subnet which would not converge quasi-uniformly and thus a contradiction.

In order to obtain the converse, assume first that the net which converges for the topology does not converge pointwise at the point $x$ in $S$. It follows that there is a subnet $\left\{f_{\beta}, \beta \in D^{\prime}\right\}$ and a convex circled neighborhood $V$ of 0 in $F^{\prime}$ such that $f_{\beta}(x)$ is not in $V$ for each $\beta$ in $D^{\prime}$. This leads to a contradiction because the set $\mathscr{G}(S, F) \sim\left\{f_{\beta}, \beta \in D^{\prime}\right\}$ has property $(\alpha)$ and the net is not eventually in it.

A similar contradiction is obtained when it is assumed that there is a subnet which converges pointwise and in the topology, but does not converge quasi-uniformly.

3. The topology of almost uniform convergence on a collection of subsets of $S$. In the above discussion the convergence has occurred over the entire set $S$. Without difficulty, the convergence can be restricted to a family of subsets of $S$.

3.1 Definition. A subset $U$ of $\mathscr{G}(S, F)$ has the property $(\alpha)$ over a subset $A$ of $S$ if it has property $(\alpha)$ in $\mathscr{C}(A, F)$, where $\mathscr{G}(A, F)$ is the linear space which is obtained by restricting the functions in $\mathscr{G}(S, F)$ to having $A$ as their domain of definition.

The analogue of Theorem 1.3 is now stated.

3.2 Theorem. Let $\mathscr{G}(S, F)$ be a linear space of function on a set $S$ into a locally convex linear topological space $F$ and let $\mathscr{A}$ be a collection of subsets of $S$. Then all the sets in $\mathscr{C}(S, F)$ of the form $U_{1} \cap \cdots \cap U_{u}$, where each $U_{i}$ has property $(\alpha)$ over some $A$ in $\mathscr{A}$, form a local base for a locally convex topology. This topology of almost uniform convergence on members of $\mathscr{A}$. Furthermore, it is a linear topology if and only if $f[A]$ is bounded for each $A$ in $\mathscr{A}$ and each $f$ in $\mathscr{C}(S, F)$ and it is a Hausdorff topology if $F$ is Hausdorff and for each $f$ in $\mathscr{C}(S, F)$ there is a point $x$ in at least one member of $\mathscr{A}$ such that $f(x) \neq 0$.

The expected analogues of the theorems in $\S 1$ are also valid. In analogy with topologies of uniform convergence it is noted that $\mathscr{A}$ can be enlarged to contain all the finite unions of its members without affecting the topology. 
4. Function spaces. It is interesting to note that the subset $\mathscr{B}$ of $\mathscr{C}(S, F)$ consisting of all functions which are bounded on each member of $\mathscr{A}$ is a closed subspace of $(S, F)$ for the topology of almost uniform convergence on members $\mathscr{\%}$. If $S$ is a topological space it is also clear that the functions which are continuous on each member of $\mathscr{\nearrow}$ form a closed subspace.

4.1 Theorem. Let $C(S, F)$ be a linear space of continuous functions on a topological space $S$ to a locally convex linear topological space $F$. Then the topology of almost uniform convergence on members of $\mathscr{\mathscr { L }}$ and the topology of almost uniform convergence on the members of a collection composed of the closures of finite unions of members of $\mathscr{A}$ are the same topology on $C(S, F)$.

Proof. Assuming that the subset $U$ of $C(S, F)$ has property $(\alpha)$ over the closure of $A$, where $A$ is in $\mathscr{Y}$, there is a closed convex circled neighborhood $V$ of 0 in $F$ such that for each finite subset $\left\{f_{1}, \cdots, f_{k}\right\}$ of $C(S, F) \sim U$ there exists an $x_{0}$ in the closure of $A$ and a neighborhood $W$ of $x_{0}$ with the property that $f_{,}(W)$ is disjoint from $V$ for $j=1, \cdots, k$. Since there is an $x$ in $W$ which is also in $A$, the proof is completed by concluding that $U$ has property $(\alpha)$ over $A$.

The following theorems give some indication of the relationship between almost uniform convergence and pointwise convergence.

4.2 Theorem. If $A$ is a compact subset of a topological space $S$ then the topology of almost uniform convergence on $A$ is equivalent to the topology of pointwise convergence on $A$ for a function space $C(S, F)$ of continuous functions defined on $S$ with range in the locally convex linear topological space $F$.

Proof. It has already been shown that almost uniform convergence implies pointwise convergence. The converse is immediately obtained by nothing that Arzelà's Theorem 2.2 establishes the quasi-uniform convergence of every subnet of a pointwise convergent net in $C(S, F)$.

Theorems 4.1 and 4.2 combine to obtain the following result.

4.3 TheOREM. Let $S$ be a completely regular Hausdorff space. The topology of almost uniform convergence on $S$ is equivalent to the topology of pointwise convergence on the Stone-Céch compactification [17] of $S$ for the function space of bounded continuous functions on $S$ with range in the complex or real numbers.

A noticeable difference between the uniform convergence topologies and almost uniform convergence topologies occurs on questions of completeness. For example, the almost uniform convergence topology on 
the linear space of all bounded real valued functions defined on the closed unit interval $[0,1]$ is not complete. If it were a complete topology the subspace of continuous function would be required to be complete in the topology of pointwise convergence (see Theorems 2.2 and 4.3).

5. Adjoint spaces and the weak topology. $[2,8,11,18,19]$. Several people have observed that almost uniform convergence is in some manner related to the adjoint space of a locally convex linear topological space. The first adjoint space is the collection of all continuous scalar valued linear functions defined on the linear topological space which is under consideration. If the adjoint space is topologized it is possible to speak of the adjoint space of the adjoint space.

The adjoint space $E^{*}$ of a locally convex linear topological space $E$ defines a natural topology on $E$ which is called the weak topology. If $E$ is considered as a collection of linear functions defined on $E^{*}$, the weak topology on $E$ is the topology of pointwise convergence on $E^{*}$.

By interchanging the roles of $E$ and $E^{*}$ in the above discussion it is seen that $E$ gives rise to a natural topology on $E^{*}$ which is called the weak* topology on $E^{*}$.

The next theorem gives a small degree of insight into the structure of the adjoint space.

5.1 THEOREM. Consider a linear space $\mathscr{G}(S, K)$ consisting of functions with domain $S$ and range in the scalar field $K$. If $\mathscr{G}(S, K)$ is given a linear topology of uniform convergence on members of a collection $\mathscr{C}$ of subsets of $S$ and $\mathscr{G}^{*}$ is the adjoint space of $\mathscr{G}(S, F)$, then there exists a natural mapping $\phi$ from the subset $\bigcup_{A \in \Re A} A$ of $S$ into $\mathscr{S}^{*}$ such that for each $x \in \bigcup_{A \in \mathscr{A}} A, \phi(x) f=f(x)$ for every $f \in \mathscr{C}(S, K)$ and for each $f^{*}$ in $\mathscr{O}^{*}$ there is an $A$ in $\mathscr{A}$ and positive scalar $\varepsilon$ with the property that $\varepsilon f^{*}$ is in the weak* closed circled convex hull of $\phi[A]$.

Proof. For each $x \in \mathbf{U}_{A \in \mathscr{A}} A, \phi(x)$ is clearly an element of $\mathscr{G}^{*}$. Considering an arbitrary $f_{0}^{*}$ in $\mathscr{C}^{*}$, let $G=\left\{f \in \mathscr{C}(S, K):\left|f_{0}^{*}(f)\right| \leq 1\right\}$. The continuity of $f_{0}^{*}$ gives a positive number $\varepsilon$ and an $A$ in $\mathscr{A}$ such that $G$ contains the neighborhood $H=\{f \in \mathscr{P}(S, K):|f(x)| \leq \varepsilon$ for all $x \in A\}$. It can be shown that the set $\left\{f^{*} \in \mathscr{G}^{*}:\left|f^{*}(f)\right| \leq \varepsilon\right.$ for each $f$ in $H$ \} which contains $\varepsilon f_{0}^{*}$ is the weak* closed circled convex hull of $\phi[A][8]$.

5.2 CoRollary. Let $E$ be a locally convex linear topological space with an adjoint space $E^{*}$. If $E^{*}$ is given the topology of uniform convergence on the bounded subsets of $E$ then the adjoint space $E^{* *}$ of $E^{*}$ is the union of the weak* closures of the images in $E^{* *}$ of the bounded subsets of $E$ under the natural mapping of $E$ into $E^{* *}$. 
In the specific case of a Banach space the results presented in the corollary were proved by M. M. Day [10] and H. H. Goldstine [13].

The weak topology on the Banach space of all continuous functions defined on the closed unit interval is finer (stronger) than the topology of pointwise convergence on the closed unit interval. Since the topology of pointwise convergence is the topology of almost uniform convergence on the closed unit interval it is clear that almost uniform convergence must be modified if it is to give the weak topology. The following definition is presented with this purpose in mind.

5.3 Definition. Let $\mathscr{G}(S, F)$ be a linear space of functions on an abstract set $S$ into a locally convex linear topological space $F$. Then a subset $U$ of $\mathscr{C}(S, F)$ has property $\beta$ over a subset $A$ of $S$ if it satisfies the following condition: for some neighborhood $V$ of 0 in $F$ it is true that for each finite subset $\left\{f_{1}, \cdots, f_{k}\right\}$ of $\mathscr{C}(S, F) \sim U$ there is a finite subset $\left\{x_{1}, x_{2}, \cdots, x_{n}\right\}$ of $A$ and a finite set of positive numbers $\left\{a_{1}, a_{2}, \cdots, a_{n}\right\}, \sum_{i=1}^{n} a_{i}=1$, such that $\sum_{i=1}^{n} a_{i} f_{j}\left(x_{i}\right)$ is not in $V$ for $j=$ $1,2, \cdots, k$.

5.4 Theorem. Consider the function space $\mathscr{C}(S, F)$. Then all sets of the form $U_{1} \cap \cdots \cap U_{n}$, where each $U_{i}$ has property $(\beta)$ over some $A$ in $\mathscr{C}$, form a local base for a locally convex topology. This is called the topology of convex almost uniform convergence on members of $\mathcal{Y}$. Furthermore, it is a linear topology if and only if $f[A]$ is bounded for each $A$ in $\mathscr{C}$ and each $f$ in $(S, F)$ and it is a Hausdorff topology if $F$ is Hausdorff and for each $f$ in $\mathscr{C}(S, F)$ there is a point $x$ in at least one member of $\mathscr{A}$ such that $f(x) \neq 0$.

The omitted proof of the above theorem is essentially the same as Theorem 2.4.

5.5 Theorem. Let $S$ be a linear topological space and let $\mathscr{L}(S, F)$ be a collection of continuous linear functions defined on $S$ with range in a locally convex linear topological space $F$. If $\cong$ is a family of subsets of $S$ such that $\mathscr{L}(S, F)$ is a linear topological space for the topology of convex almost uniform convergence on members of $\mathscr{S}$ and if $\mathscr{Y}^{\prime}$ is the collection of closed convex hulls of finite unions of members of $\mathscr{A}$, then the topology of almost uniform convergence on the members of $\mathscr{A}^{\prime}$ is the same topology.

Proof. In collaboration with Theorem 4.1 it is sufficient to show that a subset $U$ of $\mathscr{L}(S, F)$ has property $(\beta)$ over a subset $A$ of $S$ if and only if it has property $(\alpha)$ on the convex hulls of $A$. Because of the linearity of the members of $U$ the result becomes apparent upon inspecting Definitions 5.3 and 1.2. 
The theorem which follows shows that it is possible to work with the weak topology on a function space without knowing anything about the first adjoint space. In many cases this avoids the necessity of obtaining a representation of the adjoint space.

5.6 THEORem. Consider a linear space $\mathscr{G}(S, K)$ consisting of functions with domain an abstract set $S$ and range in the scalar field $K$. If $\mathscr{C}(S, K)$ is given a linear topology of uniform convergence on the members of a collection $\mathscr{A}$ of subsets of $S$, then the weak topology on $\mathscr{C}(S, K)$ is the topology of convex almost uniform convergence on members of $\mathscr{A}$.

Proof. Considering $\mathscr{C}(S, K)$ as a collection of scalar valued functions defined on $\mathscr{G}^{*}$, the weak topology on $\mathscr{C}(S, K)$ is the topology of pointwise convergence on the union of the weak* closed circled convex hulls of the collection $\{\phi[A]: A \in \Omega)$ (see Theorem 5.1). The weak* closed circled convex hull of each $\phi[A]$ is weak* compact because it is the polar of a neighborhood of 0 in $\mathscr{C}(S, K)[8]$. Since each member of $\mathscr{G}(S, K)$ is a weak* continuous function on $\mathscr{G}^{*}[8]$, the weak topology is the topology of convex almost uniform convergence on the collection $\{\phi[A]: A \in \mathscr{M}\}$ (see Theorems 5.5 and 4.2), which in turn is the topology of convex almost uniform convergence on members of $\mathscr{A}$.

5.7 CoRollary. If $E$ is a locally convex linear topological space and the first adjoint space $E^{*}$ is given the strong topology, then the weak topology on $E^{*}$ is the topology of almost uniform convergence on the bounded subsets of $E$.

Further results of this type can be obtained for a function space or an operator space whose range is contained in a locally convex linear topological space.

The above relationship of the weak topology to an almost uniform convergence topology again displays the restrictive nature of a completeness requirement on an almost uniform convergence topology [16].

In the case where $S$ is a linear space, a topology of almost uniform convergence on the members of $\mathscr{A}$ is not always equivalent to the topology af almost uniform convergence on the convex hulls of the members of $\mathscr{A}$. To clarify this point consider the weak topology on a locally convex linear topological space $E$. It is the topology of almost uniform convergence on the weak* compact subsets of the first adjoint space $E^{*}$ (see Theorem 4.2) If the weak topology on $E$ was also the topology of almost uniform convergence on the convex hulls of the weak* compact subsets of $E^{*}$ the topology of uniform convergence on the same subsets 
would be a linear topology for $E$ with the same adjoint space. This leads to contradiction in cases where the new topology would be properly finer than the Mackey topology on $E[18,19]$.

In the case of a normed linear space the following theorem due to G. Sirvint [21] is of interest. The original form of the theorem concerns sequences and not nets.

5.8 Theorem. Let $F$ be a normed linear space. A net $\left\{f_{\alpha}, \alpha \in D\right\}$ in the function space $(S, F)$ converges almost uniformly to $f_{0}$ on a subset $A$ of $S$ if and only if $\lim _{\alpha} \lim _{\beta}\left\|f_{\alpha}\left(x_{\beta}\right)-f_{0}\left(x_{\beta}\right)\right\|=0$ for every net $\left\{x_{\beta}, \beta \in D^{\prime}\right\}$ in $A$.

In Banach's book [5] on page 134 there is a necessary and sufficient condition for a sequence from the Banach space of all continuous functions on the closed unit interval to converge weakly. This theorem does not hold in the case of nets. It is now possible to state a similar theorem for nets by making use of Theorems 5.6 and 5.8. Let $\Xi$ be the collection of all sets consisting of a finite number of ordered pairs $\left\{\left(a_{1}, t_{1}\right),\left(a_{2}, t_{2}\right), \cdots,\left(a_{n}, t_{n}\right)\right\}$ of numbers from the closed unit interval $[0,1]$ with the property that $\sum_{i=1}^{n} a_{i}=1$. For each $\psi_{r} \varepsilon \Xi, \psi=\left\{\left(a_{1}, t_{1}\right)\right.$, $\left.\left(a_{2}, t_{2}\right), \cdots,\left(a_{n}, t_{n}\right)\right\}$, and each real valued function $f$ on the closed unit interval define $\psi r(f)$ to be $\sum_{i=1}^{n} a_{i} f\left(t_{i}\right)$. With this notation the theorem can be stated.

5.9 Theorem. A net $\left\{f_{\alpha}, \alpha \in D\right\}$ from the Banach space $C[0,1]$ converges weakly to $f_{0}$ if and only if $\lim _{\alpha} \underline{\lim }_{\beta}\left|\psi_{\beta}\left(f_{\alpha}\right)-\psi_{\beta}\left(f_{0}\right)\right|=0$ for every net $\left\{\psi_{\beta}, \beta \in \mathscr{P}\right\}$ in $\Xi$.

A similar theorem is available for the Banach space $L^{1}$. Let $\mathscr{C}$ be the collection of all measurable sets from the measure space upon which the function of $L^{1}$ are defined. The norm $\|f\|=\sup _{M \in M}\left|\int_{E} f d \mu\right|$ is topologically equivalent to the usual norm for $L^{1}$. If $L^{1}$ is viewed as a collection of functions defined on $\mathscr{C}$ with its topology determined by the new norm, it satisfies the hypothesis of Theorem 5.6.

Let $\Phi$ be the collection of all sets consisting of a finite number of ordered pairs $\left\{\left(a_{1}, M_{1}\right),\left(a_{2}, M_{2}\right), \cdots,\left(a_{n}, M_{n}\right)\right\}$ where $M_{i}(i=1,2, \cdots, n)$ is a measurable set from the measure space and $\left(a_{1}, a_{2}, \cdots, a_{n}\right)$ is a set of possitive numbers with the property $\sum_{i=1}^{n} a_{i}=1$. For each $q \in \Phi, \mathcal{P}=$ $\left\{\left(a_{1} M_{1}\right), \cdots,\left(a_{n}, M_{n}\right)\right\}$, and for each $f \in L^{1}$ define $\varphi(f)$ to be $\sum_{i=1}^{n} a_{i} \int_{M_{i}} f d \mu$.

This notation makes it possible to state the following theorem.

5.10 Theorem. A net $\left\{f_{\alpha}, \alpha \in D\right\}$ from the Banach space $L^{1}$ converges weakly to an element of $L^{1}$ if and only if $\lim _{\alpha} \lim _{\beta} \mid \mathcal{P}_{\beta}\left(f_{\alpha}\right)-\mathscr{P}_{\beta}\left(f_{0} \mid=0\right.$ for every net $\left\{\varphi_{\beta}, \beta \in \mathscr{B}\right\}$ in $\Phi$. 
A related theorem for sequences can be found in reference [12; page 89].

\section{BIBLIOGRAPHY}

1. L. Alaoglu, Weak topologies of normed linear spaces, Ann. Math 41 (1940), 252-267.

2. Richard Arens, Duality in linear spaces, Duke Math. J., 14 (1947), 787-794.

3. C. Arzelà, Intorno alla continuità della somma d'infinità di funzioni continue Rend. Dell'Accad. di Bologna (1883-1884), 79-84.

4. - Sulle funzioni di linee, Mem. dell'Accad. di Bologna Ser. V, 5 (1885), 225-244.

5. S. Banach, Théorie des opérations linéaires, Warsaw, 1932.

6. R. G. Bartle, On compactness in functional analysis, Trans. Amer. Math. Soc. 79 (1955), 35-37.

7. N. Bourbaki, Espaces vectoriels topologique, Chap. I-II, Actualités Scientifiques et Industrielles, no. 1189, Paris, Hermann, 1953.

8. - - Espaces vectoriels topologique, Chap. III. IV, V, Actualites Scientifiques et Industrielles, no. 1229, Paris, Hermann, 1955.

9. J. W. Brace, Almost uniform convergence, Portugalie Mathematica, 14 (1955), 99-104.

10. M. M. Day, A property of Banach spaces, Duke Math. J., 8 (1941), 763-770.

11. J. A. Dieudonné, La dualité dans les espaces vectoriels topologiques, Annales Scientifiques de L'Ecole Normale Supérieur, 59 (1942) 107-139.

12. - Sur les espaces de Köthe, Journal d'Analyse Mathématique, 1 (1951), 81-115.

13. H. H. Goldstine, Weakly complete Banach spaces, Duke Math. J., 4 (1938), 125-131.

14. A. Grothendieck, Sur les applications linéaires faiblemeni compactes d'espace du type $C(K)$, Can J. Math., 5 (1953), 129-173.

15. D. H. Hyers, Linear topological spaces, Bull. Amer. Math. Soc., 51 (1945), 1-21.

16. Samuel Kaplan, Cartesian products of reals, Amer. J. Math., 74 (1952), 936-954.

17. J. L. Kelley, General topology, Van Nostrand Co., New York, 1955.

18. G. W. Mackey, On infinite dimensional linear spaces, Trans. Amer. Math. Soc., 57 (1945), 155-207.

19. - - On convex topological linear sraces, Trans. Amer. Math. Soc., 60 (1946), 519-537.

20. J. von Neumann, On complete topological spaces, Trans. Amer. Math. Soc., 37 (1935), $1-20$.

21. G. Sirvint, Weak compactness in Banach spaces, Studia Mathematica, 6 (1957), 71-94.

22. J. V. Wehausen, Transformations in linear topological spaces, Duke., Math J., 4 (1938), 157-169.

UNIVERSITY OF MARYLAND 


\section{PACIFIC JOURNAL OF MATHEMATICS}

\section{EDITORS}

\section{David Gilbarg}

Stanford University Stanford, California

\section{R. A. Beaumont}

University of Washington

Seattle 5, Washington

\section{A. L. Whiteman}

University of Southern California Los Angeles 7, California

L. J. Paige

University of California

Los Angeles 24, California

\section{ASSOCIATE EDITORS}

E. F. BECKENBACH
C. E. BURGESS
E. HEWITT
A. HORN

A. HORN

\author{
V. GANAPATHY IYER \\ R. D. JAMES \\ M. S. KNEBELMAN \\ L. NACHBIN
}

I. NIVEN

T. G. OSTROM

H. L. ROYDEN

M. M. SCHIFFER
E. G. STRAUS

G. SZEKERES

F. WOLF

K. YOSIDA

\section{SUPPORTING INSTITUTIONS}

\author{
UNIVERSITY OF BRITISH COLUMBIA \\ CALIFORNIA INSTITUTE OF TECHNOLOGY \\ UNIVERSITY OF CALIFORNIA \\ MONTANA STATE UNIVERSITY \\ UNIVERSITY OF NEVADA \\ OREGON STATE COLLEGE \\ UNIVERSITY OF OREGON \\ OSAKA UNIVERSITY \\ UNIVERSITY OF SOUTHERN CALIFORNIA
}

\author{
STANFORD UNIVERSITY \\ UNIVERSITY OF TOKYO \\ UNIVERSITY OF UTAH \\ WASHINGTON STATE COLLEGE \\ UNIVERSITY OF WASHINGTON \\ * * * * \\ AMERICAN MATHEMATICAL SOCIETY \\ CALIFORNIA RESEARCH CORPORATION \\ HUGHES AIRCRAFT COMPANY \\ SPACE TECHNOLOGY LABORATORIES
}

Mathematical papers intended for publication in the Pacific Journal of Mathematics should be typewritten (double spaced), and the author should keep a complete copy. Manuscripts may be sent to any one of the four editors. All other communications to the editors should be addressed to the managing editor, L. J. Paige at the University of California, Los Angeles 24, California.

50 reprints per author of each article are furnished free of charge; additional copies may be obtained at cost in multiples of 50 .

The Pacific Journal of Mathematics is published quarterly, in March, June, September, and December. The price per volume (4 numbers) is $\$ 12.00$; single issues, $\$ 3.50$. Back numbers are available. Special price to individual faculty members of supporting institutions and to individual members of the American Mathematical Society: $\$ 4.00$ per volume; single issues, $\$ 1.25$.

Subscriptions, orders for back numbers, and changes of address should be sent to Pacific Journal of Mathematics, 2120 Oxford Street, Berkeley 4, California.

Printed at Kokusai Bunken Insatsusha (International Academic Printing Co., Ltd.), No. 6, 2-chome, Fujimi-cho, Chiyoda-ku, Tokyo, Japan.

PUBLISHED BY PACIFIC IOURNAL OF MATHEMATICS, A NON-PROFIT CORPORATION

The Supporting Institutions listed above contribute to the cost of publication of this Journal, but they are not owners or publishers and have no responsibility for its content or policies. 


\section{Pacific Journal of Mathematics}

\section{Vol. 9, No. $3 \quad$ July, 1959}

Errett Albert Bishop, A minimal boundary for function algebras . . . . . . . . . . . . 629

John W. Brace, The topology of almost uniform convergence . . . . . . . . . . . . 643

Cecil Edmund Burgess, Chainable continua and indecomposability .......... 653

L. Carlitz, Multiplication formulas for products of Bernoulli and Euler

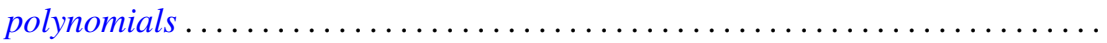

Eckford Cohen, A class of residue systems (mod $r$ ) and related arithmetical

functions. II. Higher dimensional analogues ....................

Shaul Foguel, Boolean algebras of projections of finite multiplicity . . . . . . . . . .

Richard Robinson Goldberg, Averages of Fourier coefficients .................

Seymour Goldberg, Ranges and inverses of perturbed linear operators .

Philip Hartman, On functions representable as a difference of convex functions ....

Milton Vernon Johns, Jr. and Ronald Pyke, On conditional expectation and

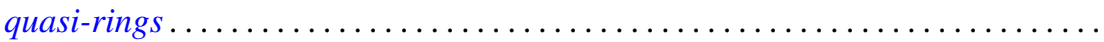

Robert Jacob Koch, Arcs in partially ordered spaces ....................

Gregers Louis Krabbe, A space of multipliers of type $L^{p}(-\infty, \infty) \ldots \ldots \ldots \ldots$

John W. Lamperti and Patrick Colonel Suppes, Chains of infinite order and their

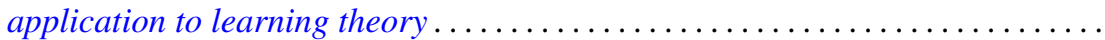

Edith Hirsch Luchins, On radicals and continuity of homomorphisms into Banach

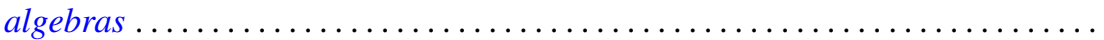

T. M. MacRobert, Multiplication formulae for the E-functions functions of their parameters.

Michael Bahir Maschler, Classes of minimal and representative domains and their kernel functions.

William Schumacher Massey, On the imbeddability of the real projective spaces in Euclidean space.

Thomas Wilson Mullikin, Semi-groups of class $\left(C_{0}\right)$ in $L_{p}$ determined by parabolic differential equations

Steven Orey, Recurrent Markov chains

Ernest Tilden Parker, On quadruply transitive groups ........ . .

Calvin R. Putnam, On Toeplitz matrices, absolute continuity, and unitary

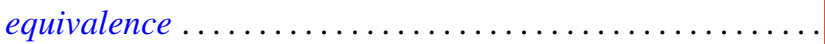

Helmut Heinrich Schaefer, On nonlinear positive operators.

Robert Seall and Marion Wetzel, Some connections between continued fractions and convex sets

Robert Steinberg, Variations on a theme of Chevalley

Olga Taussky and Hans Zassenhaus, On the similarity transformation between a

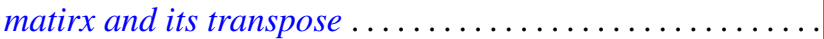

Emery Thomas, The suspension of the generalized Pontrjagin cohomology

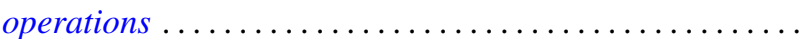

Joseph L. Ullman, On Tchebycheff polynomials ..................... 913

Richard Steven Varga, Orderings of the successive overrelaxation scheme ........ 925

Orlando Eugenio Villamayor, Sr., On weak dimension of algebras . 